\title{
ACCESO A LA ATENCIÓN ODONTOLÓGICA E INEQUIDAD EN EL PERÚ EN EL AÑO 2015
}

\section{ACCESS TO DENTAL ATTENTION AND INEQUITY IN PERU 2015}

\author{
Alarcón-Cruzado Ricardo. ${ }^{1 *}$ León-Manco Roberto Antonio $^{2}$ \\ ${ }^{1}$ Cirujano dentista.Universidad Peruana Cayetano Heredia.Perú. \\ ${ }^{2}$ Maestro en Salud Pública.Departamento Académico de Odontología Social, Unidad de Investigación en Salud Pública y \\ Gestión Estomatológica, Universidad Peruana Cayetano Heredia. Perú. \\ *ricardo.alarcon@upch.pe
}

\begin{abstract}
Resumen
OBJETIVO Determinar el de acceso a la atención odontológica e inequidad en niños de 0 a 11 años y adultos mayores de 60 años de edad, según ámbito geográfico en el Perú en el año 2015. Materiales y métodos: Se utilizaron la Encuesta Demográfica y de Salud Familiar (ENDES) del año 2015 y Medición de pobreza según Cifras de pobreza en el Perú (INEI; 2015). El estudio fue de tipo descriptivo, transversal y ecológico. Las variables del estudio fueron acceso a servicios odontológicos, inequidad, espacio geográfico y región natural. Se ejecutó un análisis descriptivo, de las variables cualitativas se obtendrán las frecuencias absolutas y relativas, las cuales se organizarán y presentarán en tablas y gráficos. Resultados: En niños de 0-11 años de edad, a nivel de ámbito geográfico, se observa que existe mayor acceso odontológico en la zona urbana (51.3\%), según región natural, se obtuvo un mayor acceso en la sierra $(52.4 \%)$ y a nivel nacional, se observa que un $47.6 \%$ tuvo acceso odontológico. En personas mayores de 60 años, a nivel de espacio geográfico, se observa que existe mayor acceso en la zona urbana $(86.8 \%)$, según región natural, se obtuvo un mayor acceso en la costa $(87.3 \%)$ y a nivel nacional, se observa que $77.3 \%$ tuvo acceso odontológico. Conclusiones: Se evidencia gráficamente una tendencia que a menor inequidad según ámbito geográfico (espacio geográfico y región natural) existe mayor acceso a la atención odontológica en niños de 0 a 11 años y personas mayores de 60 años de edad.
\end{abstract}

Palabras clave: Inequidad, acceso a servicios de salud, odontología .

\begin{abstract}
OBJECTIVE Determine the access to dental care according to inequality by geographical area in Peru in 2015. Materials and methods: The Demographic and Family Health Survey (ENDES) was used in 2015 and Measuring poverty according to Poverty figures in Peru (INEI, 2015). The study was descriptive, transversal and ecological. The variables of the study were access to dental services, inequality, geographic space and natural region. A descriptive analysis was performed, qualitative variables will be obtained the absolute and relative frequencies, which will be organized and presented in tables and graphs. Results: In children of 0-11 years of age, in the geographical area, it is observed that there is greater odontological access in the urban area (51.3\%), according to natural region, greater access was obtained in the highlands (52.4\%), and at the national level, $47.6 \%$ had dental access. In people older than 60 years of age, at the level of geographic space, it is observed that there is greater access in the urban area (86.8\%), according to natural region, greater access was obtained at the coast (87.3\%) and at national level, that $77.3 \%$ had dental access. Conclusions: There is a graphical evidence of a trend towards greater inequality according to geographic area (geographical area and natural region). There is greater access to dental care in children aged 0 to 11 years and people over 60 years of age.
\end{abstract}

Key words: Inequity, access to health services, dentistry. .

\section{INTRODUCCIÓN}

La inequidad social como concepto constituye la diferencia entre poblaciones o grupos que conforman una sociedad, siendo una de las causas la desigualdad de oportunidades para acceder a bienes y servicios como: educación, vivienda o salud, las que a su vez pueden ser consecuencias a este problema. ${ }^{1}$ Esta situación desfavorable afecta a la gran mayoría de la población, haciendo difícil su acceso a los servicios de salud. Desde hace algunas décadas, este tema se ha ido investigando, siendo uno de los temas que ha despertado 
interés en el área de salud. Por otra parte, en el ámbito odontológico, los estudios se han centrado en determinar qué factores influyen en las personas para decidir visitar al dentista. ${ }^{2}$

En diferentes países, tanto de ingresos altos, medios o bajos, se ha evidenciado que los factores sociales, como la situación laboral, educación, el nivel de ingresos, la raza y el sexo, tiene una alta influencia en la salud de los diferentes conjuntos sociales. Cuanto más inferior es el nivel socioeconómico de una persona, mayor es el riesgo de no obtener una buena calidad en los servicios de salud. Existen muchos centros poblados dentro del Perú que el acceso a la atención odontológica es complicado, debido a la falta de conocimientos de los pobladores, así como, la falta de comunicación del personal de salud. ${ }^{3}$

La Organización Mundial de la Salud (OMS) en su Comisión sobre Determinantes Sociales de la Salud, ${ }^{4}$ resaltó que no han existido estrategias fundamentales para mermar la inequidad y a su vez prevenir enfermedades graves no transmisibles. Cabe destacar que las patologías orales son las más prevalentes de las enfermedades crónicas, y a su vez, las más resaltantes en los problemas de salud pública, debido a su alta incidencia en distintas comunidades, y por el alto costo de su tratamiento. ${ }^{5}$

En una revisión sistemática, Moreira et al, ${ }^{6}$ hallaron como las más importantes barreras que perjudican al acceso de servicios odontológicos, el bajo nivel de educación, deficiencia de recursos económicos y la escasa promoción de servicios públicos de atención en salud bucal para las poblaciones que desconocen estas mismas. La desigualdad entre los diferentes niveles sociales, comprende uno de los factores que está asociado de manera directa y en forma más sistemática con los daños en salud. Es muy probable que esta desigualdad socioeconómica seguirá estando en las próximas décadas y no solamente presente hoy en día. Por ello, el sistema de salud debe buscar la equidad enfocando sus recursos hacia donde existan mayores necesidades y menor acceso a los servicios.

En el Perú está evidenciado que la falta de uso de los servicios de salud se encuentra directamente relacionado al pobre nivel de educación de la persona y a su vez al poco ingreso económico que pueda tener. ${ }^{4}$ Adicionalmente, existen otras variables que influencian en la decisión de las personas en acudir a un centro de salud y recibir atención, tales como: ubicación geográfica, ocupación, aspectos culturales, actitudes frente a la salud, experiencias previas de atención, entre otros. 8,9

Por ello, el objetivo del estudio fue determinar el acceso a la atención odontológica según inequidad por ámbito geográfico en el Perú en el año 2015.

\section{MATERIALES Y MÉTODOS}

El presente estudio fue ecológico, la muestra estuvo constituida por los registros datos del Instituto Nacional de
Estadística e Informática del Perú (INEI), para el caso de acceso a atención odontológica se empleó los registros de 47443 niños de 0 a 11 años y 4370 de las personas mayores de 60 años de edad pertenecientes a la Encuesta Demográfica y de Salud Familiar (ENDES) del año 2015. Para el caso de inequidad se emplearon los datos de la medición de la pobreza del Informe Técnico: Evolución de la Pobreza Monetaria del Perú del INEI del mismo año.

$\mathrm{El}$ acceso a servicios odontológicos fue medido mediante la frecuencia relativa en niños de 0 a 11 años de edad y en mayores de 60 años de edad, estos grupos son en los cuales se levantan los datos. La inequidad fue medida mediante el Coeficiente de Gini proporcionado por el INEI como parte de la medición anual de la pobreza en el país. Ambas variables fueron organizadas por ámbito geográfico, la cual tiene dos dimensiones: espacio geográfico considerando el espacio rural y urbano, y, región natural siendo costa, sierra y selva.

El primer paso para la realización de la presente investigación fue acceder a la base de datos de datos de la Encuesta Demográfica y de Salud Familiar (ENDES) del año 2015, disponible de forma libre en la página web oficial (www.inei.gob.pe), se accedió al link "Bases de datos", luego se abrió el enlace "Microdatos", seguido "Consulta por Encuestas", y en el buscador se seleccionó ENDES 2015. A continuación se accedió a la base de datos del Informe Técnico: Evolución de la Pobreza Monetaria 2009-2015, disponible de forma libre en la página web oficial (www.inei.gop.pe); se abrió el link "Estadísticas", luego cifras de pobreza año 2015 , seguido por Informe Técnico. Posteriormente se descargó completamente la base de datos en formato SPSS, con esa base se seleccionaron las preguntas de acceso a servicios de salud bucal en la población de niños de 0-11 años y la población de adultos mayores de 60 años a más, específicamente las preguntas 803 y 311 respectivamente, adicionando las variables de inequidad, espacio geográfico y región natural. Del Informe Técnico de Evolución de la Pobreza Monetaria 2009-2015, se obtuvo el coeficiente de Gini según ámbito geográfico, el cual se encuentra de acceso libre en la misma web mencionada.

El presente estudio fue de tipo descriptivo, de las variables cualitativas se obtuvieron las frecuencias absolutas y relativas, las cuales se organizaron y presentaron en tablas y gráficos. Los datos fueron analizados utilizando el programa estadístico SPSS v. 24.0 y Excel 2013.

\section{RESULTADOS}

Según la base de datos de la Encuesta Demográfica y Salud Familiar (ENDES) 2015, se tuvo un registro de 47443 niños de 0 a 11 años y 4370 de las personas mayores de 60 años de edad, organizado por espacio geográfico y región natural.

Para niños de 0 a 11 años, según ámbito geográfico, se obtuvo que en la zona urbana existe mayor acceso odon- 
tológico con $51.3 \%(\mathrm{n}=15784)$ con menor inequidad (IG: $0.40)$ y en la zona rural existe un menor acceso odontológico $40.9 \%(n=6822)$ con mayor inequidad (IG: 0.41). Por región natural, se observa un mayor acceso en la zona de la sierra con $52.4 \%(\mathrm{n}=8499)$ con mayor inequidad (IG: 45.00), seguido por la costa con $51.3 \%(\mathrm{n}=9400)$, inequidad (IG: $0.40)$ y la selva con menor acceso odontológico con $36.5 \%$ $(\mathrm{n}=4707)$ y mayor inequidad (IG: 0.46). A nivel nacional, se observa un $47.6 \%(n=22606)$ que tuvieron acceso a servicios odontológicos con inequidad (IG: 0.44) (Tabla 1 y Gráfico N $1)$.

\begin{tabular}{|c|c|c|c|c|}
\hline \multirow{2}{*}{\multicolumn{2}{|c|}{ AMBITO GEOGRÁFICO }} & \multirow{2}{*}{$\begin{array}{c}\text { Inequidad } \\
\text { Îndice de Gini }\end{array}$} & \multicolumn{2}{|c|}{ Acceso de 0 a 11 años } \\
\hline & & & $\mathbf{n}$ & $\%$ \\
\hline \multicolumn{5}{|c|}{ Espacio geográfico } \\
\hline & Urbana & 0.40 & 15784 & 51.3 \\
\hline & Rural & 0.41 & 6822 & 40.9 \\
\hline \multicolumn{5}{|c|}{ Región natural } \\
\hline & Costa & 0.40 & 9400 & 51.3 \\
\hline & Sierra & 0.45 & 8499 & 52.4 \\
\hline & Selva & 0.46 & 4707 & 36.5 \\
\hline Nacional & & 0.44 & 22606 & 47.6 \\
\hline
\end{tabular}

n: Frecuencia absoluta.

$\%$ : Frecuencia relativa.

Tabla 1. Acceso a la atención odontológica en niños de 0 a 11 años según inequidad por espacio geográfico y región natural en el Perú, 2015.

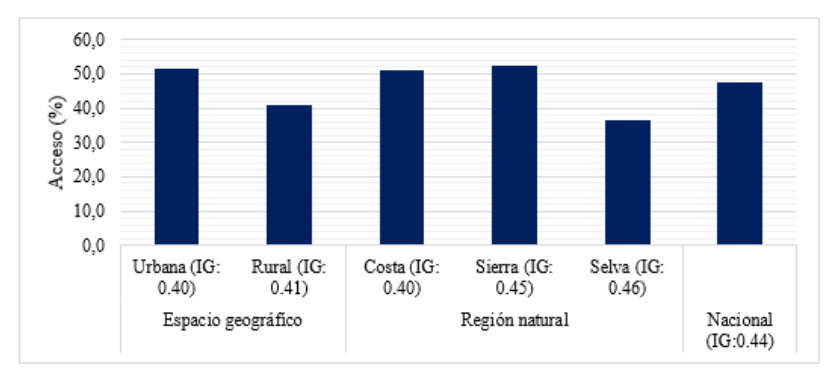

Grf. 1. Acceso a la atención odontológica en niños de 0 a 11 años según inequidad por espacio geográfico y región natural en el Perú, 2015.

Para personas mayores a 60 años, según ámbito geográfico, se obtuvo que en la zona urbana existe mayor acceso odontológico $86.8 \%(\mathrm{n}=2190)$ con menor inequidad (IG: 0.40 ), mientras que en la zona rural existe menor acceso odontológico $64.4 \% \quad(\mathrm{n}=1190)$ con mayor inequidad (IG: 0.41). Por región natural, se observa un mayor acceso en la zona de la costa $87.3 \%(n=1493)$ con menor inequidad (IG: 0.40$)$, seguido por la sierra $71.3 \%(n=1332)$, inequidad (IG: 0.45 ) y la selva con menor acceso odontológico $70.2 \%$ $(\mathrm{n}=555)$ y mayor inequidad (IG: 0.46). A nivel nacional, se observa un $77.3 \%(n=3380)$ que tuvieron acceso a servicios odontológicos con inequidad (IG: 0.44) (Tabla 2 y Gráfico N 2).

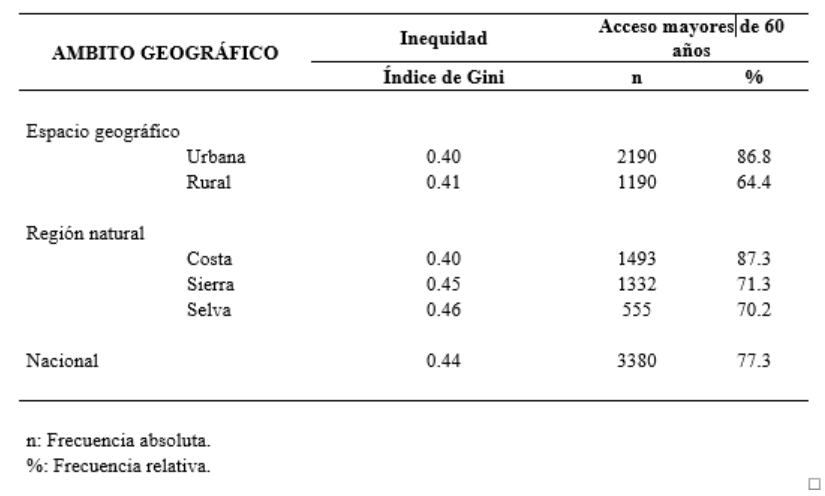

Tabla 2. Acceso a la atención odontológica en personas mayores de 60 años según inequidad por espacio geográfico y región natural en el Perú, 2015.

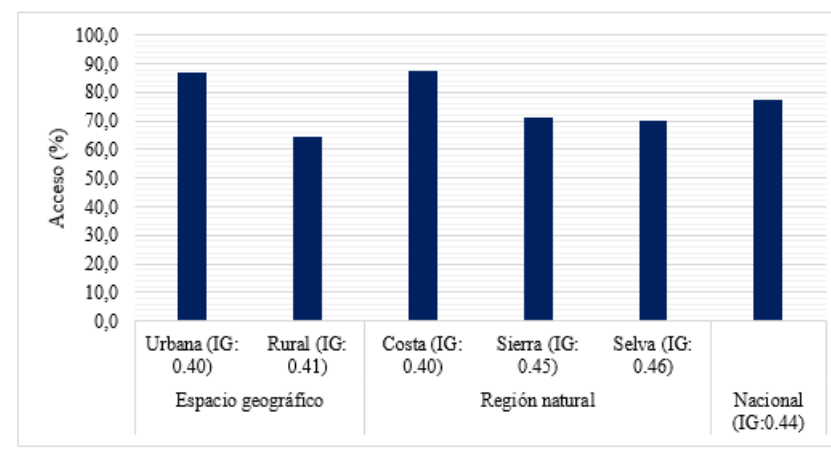

Grf. 2. Acceso a la atención odontológica en personas mayores de 60 años según inequidad por espacio geográfico y región natural en el Perú, 2015.

\section{DISCUSIÓN}

La equidad en salud es un concepto con un estudio bastante complejo, porque existen diferentes filosofías, partiendo desde lo socioeconómico y el estado de salud de un grupo de personas, que la definen como tal. ${ }^{10}$ Desde una perspectiva de justicia social, se dice que todas las personas deben tener la misma oportunidad de lograr su estabilidad en salud y que ninguna debería estar en desventaja al momento de intentar conseguirla. La equidad no significa tener el mismo nivel en salud, ni tener acceso a los mismos servicios y recursos, esto va a depender del contexto de necesidad de cada persona según lo requiera. ${ }^{11}$

Según la Comisión sobre Determinantes Sociales de la Salud de la OMS (CDSS), ${ }^{12}$ afirma que la mala distribución del poder, ingresos, bienes y servicios, son los que afectan a las condiciones de vida a nivel nacional y mundial. Por 
consiguiente, todo aquello que las personas tengan como necesidad para su desarrollo, tales como educación, economía, vivienda y salud, se mostrarán alteradas.

Para Amartya Sen, ${ }^{13}$ la equidad debe ser comprendida desde distintas dimensiones, para poder entender la justicia social. El autor introduce un concepto distinto al considerar el problema de la justicia sanitaria y de la equidad en salud particularmente en relación a las capacidades de las personas, al desarrollo social, económico y cultural.

Todos estos conceptos enfatizan el problema de inequidad en varios aspectos que lleva a una desigualdad e injusticia en la población para obtener una calidad de vida próspera. Las personas que viven en la pobreza, escasos grupos étnicos u otros grupos que no han desarrollado una economía aceptable, no tienen la oportunidad o es muy complicado para ellos acceder a servicios de salud, este problema afecta especialmente a niños, mujeres y adultos mayores. En el caso de las mujeres con menor rango de ingresos y de acceso a servicios de planificación y salud, corren el riesgo de quedar embarazadas a muy temprana edad, lo que implica que esto perjudicaría directamente a sus hijos, porque muchas de ellas no manejan suficiente información prenatal adecuada, o no es atendida por un profesional capacitado al momento de dar a luz, todo esto sumado con el nivel de educación de la mujer, dificultan la probabilidad de supervivencia, tanto para ellas mismas, como para los niños. ${ }^{14}$

Existen diferentes investigaciones a nivel mundial que hablan acerca de cómo la inequidad es un factor determinante que dificulta el acceso a los servicios de salud y hasta muchas veces lo hace imposible, tomando en consideración los recursos de cada población por ámbito geográfico en el que residen.

En el estudio "Oral health in America: A report of the surgeon general", ${ }^{15}$ los niños en Estados Unidos que viven en la pobreza, son los más afectados por las caries, que es la enfermedad crónica infantil más común en este país, pasando por encima del asma y la fiebre. Este problema de salud oral se va acumulando hasta llegar a la adultez, trayendo como consecuencia muchas veces la pérdida de sus dientes. Varios de estos problemas se deben a: tener un nivel socioeconómico bajo, ser desempleado o pertenecer a un grupo étnico minoritario, como por ejemplo en el caso de los adultos mayores, esta parte de la población no tiene un acceso a servicios de salud ideal y muchos de ellos están en desventaja al no estar afiliado a un seguro de salud para su atención, por lo que la inequidad para ellos incrementa no consiguiendo un tratamiento adecuado e inmediato. ${ }^{16}$ Estudios acerca de inequidad en España indica una mejora en el acceso a servicios de salud por parte de la población que se encuentra adversa a obtener estos beneficios, específicamente un estudio en Cataluña; indica que el $34.3 \%$ de la población tuvo atención por un odontólogo en años previos al 2002, la mejoría se empezó a notar desde el año 1994, debido a que las personas contaron con un seguro privado y público para la atención de la salud bucal.43 Sin embargo, si comparamos con otros países de Europa, tales como Suecia y Dinamarca que bordea el $80 \%$ de personas que tienen acceso a servicios de salud o el Reino Unido que el porcentaje llega a $47 \%$, se observa que la prevalencia de acceso es baja. En otros países, de este mismo continente como Grecia y Portugal, mostraron resultados entre $23,3 \%$ y $28,8 \% .^{17,18}$

En Latinoamérica, precisamente Colombia, existe el fenómeno de desplazamiento de personas, el cual es muy común en este país, lo que causa inequidad en el acceso a servicios de salud. Entre el 2006 y el 2007 se registraron alrededor de 3000 desplazamientos, esto se ve reflejado en el índice de Gini $(58,6)$, pero en comparación con otros países como Argentina $(51,3)$ y Ecuador $(48,2)$, el resultado de inequidad es mucho mayor. ${ }^{19}$ En el presente estudio, para niños de 0 a 11 años de edad y para adultos de 60 años en adelante, a nivel nacional se presenta un índice de Gini (44.00). Mucho tiene que ver la distribución equitativa de los bienes, así como también de las riquezas de cada país, esto es porque no necesariamente el que tiene más economía, tiene mejor beneficio en la salud.

Según la Comisión sobre los Determinantes de la Salud de la OMS (CDSS), ${ }^{20}$ menciona que las riquezas por sí solas no es un factor determinante para mejorar la salud de un país. Algunos países con ingresos muy bajos como Cuba, Costa Rica, China o la India, han logrado obtener un nivel en salud bastante bueno, aplicando estrategias como: equidad de género, alentar la igualdad de acceso a servicios y beneficios, misma oportunidad de trabajo para las personas y bajos niveles de exclusión social, sin duda una estrategia bastante notable que se debe aplicar para mejorar la equidad en salud de otros países. Por consiguiente, si se logra tener una repartición inteligente de los distintos factores de acceso a servicios, disminuirá el porcentaje de inequidad en materia de salud.

Según Hernández et al (Perú; 2014), explora los patrones de acceso a servicios de salud dental en menores de doce años en el Perú, encontrando que el acceso fue de $40.6 \%$ zona rural, $58.3 \%$ zona urbana, ${ }^{21}$ en la presente investigación se realizó según la base de datos de la Encuesta Demográfica y Salud Familiar (ENDES) 2015, donde se tuvo un registro de 47443 niños de 0 a 11 años, siendo los resultados según ámbito geográfico; $51.3 \%$ en zona urbana $(\mathrm{n}=15784)$ con menor inequidad (IG: 40.00$)$ y $40.9 \%$ en zona rural $(n=6822)$ con mayor inequidad (IG:41.00). Haciendo una similitud entre uno y otro estudio, tomando en cuenta que son de años consecutivos, existe un porcentaje aún bajo en la zona rural, lo que nos indica la falta de promoción de salud y concientización de la población para acceder a los servicios odontológicos, mientras que en la zona urbana ocurre lo contrario, se evidenció un aumento en el porcentaje de personas que acudieron a estos servicios, ya que en este sector de la población el nivel socioeconómico desempeña un rol determinante y distintos seguros en los que la población 
se encuentra afiliada.

Para los resultados de niños de 0-11 años de edad según región natural se tiene que en la Costa $(n=9400)$ es mayor que es de la Sierra $(n=8499)$, con inequidades de IG: 0.40 y 0.45 respectivamente, para este caso tenemos una alteración en los valores, porque que el $\mathrm{N}$ de la costa es mayor pero con menor porcentaje de acceso $(51,3 \%)$ que el de la sierra, mientras que este último tiene mayor porcentaje de acceso $(52.4 \%)$, pero el $\mathrm{N}$ es menor. Esta eventualidad es porque en el año 2015 el Programa de Salud escolar fue aplicado con mayor énfasis en la zona de la sierra para poder promover prácticas y conductas para el cuidado de la salud. No solo el programa está dirigido a salud bucal, sino a otras áreas como nutrición, tamizaje de agudeza visual, dosaje de hemoglobina con tratamiento de anemia y salud mental. Para este último se inició un piloto en las zonas de: Apurímac, Moquegua, Arequipa, Ucayali, Callao, La Libertad y Lima (Carabayllo e Independencia). ${ }^{22}$

Para Azañedo et al (Perú, 2016), da a conocer patrones de acceso a servicios de salud dental, según área de residencia, región natural, tiempo desde la última atención y lugar de atención de los adultos mayores en el Perú, encontrando que Arequipa fue el de mayor acceso $(34.5 \%)$ y Cajamarca el de menor acceso $(10.2 \%),{ }^{23}$ en la presente investigación según la base de datos de la Encuesta Demográfica y de Salud Familiar, se obtuvo un registro de 4370 de personas mayores de 60 años de edad, siendo los resultados: según ámbito geográfico, zona urbana $86.8 \%(\mathrm{n}=2190)$ con menor inequidad (IG:40.00) y zona rural $64.4 \%(\mathrm{n}=2190)$ con mayor inequidad (IG:41.00). Según región natural, costa $87.3 \%(\mathrm{n}=1493)$ con inequidad (IG: 40.00), sierra $71.3 \%$ $(n=1332)$ con inequidad (IG: 45.00), selva $70.2 \%(n=555)$ con inequidad (IG: 46.00).

Es importante recalcar que, la gran mayoría de tratamientos para los adultos mayores es la parte protésica (prótesis parcial removible, prótesis total), estos tratamientos no están cubiertos por un seguro, por lo que las personas acuden a una atención privada donde se realiza el procedimiento de manera adecuada. Esto hace referencia al mayor porcentaje de acceso en la zona de la costa, por los distintos factores que hacen posible la atención (nivel socioeconómico, consultorios dentales privados, conocimiento del paciente), mientras que en la zona de la sierra es un poco contradictorio los resultados entre acceso e inequidad, porque siguiendo una línea lógica de las tres regiones naturales se obtuvo que: a menor inequidad, mayor acceso y a mayor inequidad, menor acceso. Esta variante en la zona de la sierra podría estar relacionado con los programas de ayuda social a los adultos mayores como "Vuelve a Sonreír", cuyo propósito es confeccionar prótesis total o removible, para las personas en situación de pobreza o extrema pobreza. Sin embargo estos tratamientos son de manera temporal y no garantiza una rehabilitación adecuada, acrecentando de esta manera la desigualdad en salud. ${ }^{24}$
El Perú, en el transcurso de su historia, ha sufrido diversos cambios en el sistema de salud por falta de una adecuada política de Estado. Entre los años 1978 y 1979 se creó el Sistema Nacional de Servicios de Salud (SNSS) en coordinación con todas las entidades en salud a través del Consejo Nacional en Salud y se obtuvieron metas importantes para lograr equidad, como prestación de salud mediante los seguros sociales, medicamentos básicos y plan nacional para la atención primaria de salud, sin embargo, este alentador comienzo fue derogado en 1990 sin previa evaluación. En este mismo año se crea el Sistema Nacional de Salud (SNS) que también fue eliminado por falta de apoyo político en el 2002. Para este último año, se crea el actual Sistema Nacional Coordinado y Descentralizado de Salud (SNCDS), el cual de la misma forma no ha tenido una sostenibilidad por parte del gobierno y ha entrado en un proceso de reforma a partir del año 2013. ${ }^{25}$

Es bastante llamativa la inestabilidad y la discontinuidad del sistema peruano a lo largo de la historia, esto hace referencia a la gran inequidad y el retraso de políticas en salud en relación con otros países del continente, queda claro que si no se continúa con un consolidado sistema, no podrá existir desarrollo ni equidad. ${ }^{26}$

A causa de todo este desequilibrio, los recursos humanos también se ven alterados: remuneraciones bajas, conflictos permanentes, insuficiencia de personal de salud en los hospitales, incluso un alto número de migración de médicos y enfermeros al extranjero. ${ }^{27}$

Otro punto a tomar en cuenta es el incremento demográfico que hay dentro de las regiones del país, esto se ha visto afectado en que muchos hospitales y/o centros de atención en salud quedan totalmente congestionados y no se dan abasto suficiente para una atención adecuada, por lo que la inequidad al acceso a servicios de salud aumenta. Gran parte de esta inequidad, lo crean los propios gobiernos de turno, porque no continúan los programas de salud ya establecidos del gobierno anterior, creando más una burocracia que eficiencia en cuanto al personal para poder llegar a satisfacer las necesidades de la población. ${ }^{27}$

Con respecto a las enfermedades de salud mental, que atacan en su mayoría a los adultos mayores, se debe a la poca importancia que se le ha brindado a la prevención y a la falta de recursos para poder atender a las zonas rurales donde no hay acceso a los servicios de salud. Lo mismo sucede en el caso de las personas que padecen de VIH y también, en el caso de los niños, con la Tuberculosis, las cuales por su alto costo de tratamiento y la deficiencia para poder hacerles llegar los medicamentos adecuados, sufren de una inequidad extrema con respecto a las poblaciones más centralistas. ${ }^{28}$

El estudio tiene limitaciones, debido a que solo se enfoca en analizar a las poblaciones por ámbito geográfico, más no a las personas de manera individual, es por ello que esta medición genera la susceptibilidad de tener un sesgo de extrapolación de resultados y no se debe caer en la falacia 
ecológica. Otra limitación del estudio es que teniendo los 10 resultados sólo genera una hipótesis de lo que está ocurriendo en cada población, más no la comprueba, para ello, se deben seguir con otros estudios transversales.

La presente investigación tiene relevancia porque muestra datos por ámbito geográfico en el Perú donde aún existe inequidad para acceder a un servicio de salud odontológica, 12 mucho de esto tiene que ver con la transmisión de los conocimientos del personal de salud a la población, para que ellos acudan a una entidad prestadora de estos servicios, no solo por un caso de emergencia, sino también para mejorar su calidad de salud bucal. Teniendo estos resultados se podrán generar estrategias donde todavía la inequidad es mayor y poder crear un plan para que cada región del Perú pueda llegar a un acceso equitativo con las distintas medidas de cada caso.

\section{CONCLUSIÓN}

Se evidencia gráficamente una tendencia que a una menor según el ámbito geográfico ( espacio geográfico y región natural) existe mayor accesos a la atención odontológica en niños de 0 a 11 años de edad y personas mayores de 60 años de edad en el Perú en el año 2015.

\section{Referencias}

1 "Inequidad" (s/f.). En significados.com. Disponible en: https://www.significados.com/inequidad/.[Consultado: 23 de abril de 2017, 10:35 am].

2 Lara N, López V, Morales S, Cortés E. Satisfacción de usuarios de dos servicios odontológicos en Tláhuaz Distrito Federal. Rev ADM. 2000; 57(2):45-9.

3 Organización Mundial de la Salud. 10 datos sobre las inequidades sanitarias y sus causas.

4 Organización Mundial de la Salud. Comisión sobre Determinantes Sociales de la Salud.

5 Sheiham A. Oral health, general health and quality of life. Bulletin of the World Health Organization 2005 Sept; 83(9): 644-644.

6 Moreira RS, Nico LS, Tomita NE, Ruiz T. A saúde bucal do idoso brasileiro: revisão sistemática sobre o quadro epidemiológico e acesso aos serviços de saúde bucal. Cad Saúde Pública. 2005; 21 (6): 1665-75.

7 Cátedra Iberoamericana. Equidad y desigualdad en salud: ¿Dos caras de la misma moneda?. Universidad de les Illes Balears.2017.

8 Miranda C, Peres M. Determinantes de la utilización de servicios dentales por los adultos: un estudio basado en la población en Florianópolis, Estado de Santa Catarina, Brasil. Cad Saude Pública. 2013; 29(11):2319- 32.

9 Núñez G, Contreras V, Correa G, Canales T, Mejía G, Oxman-Martínez J, Moreau J. Factores asociados a la consulta odontológica en niños/as y jóvenes de Talca (Chile) e inmigrantes chilenos de Montreal (Canadá). Gac Sanit. $2013 ; 27(4): 344-9$.
10 Meter F, Evans T. Dimensiones éticas de la equidad. En: Evans y colb, editores. Desafío a la falta de equidad en salud. Washington DC: Fundación Rockefeller/Organización Panamericana de la Salud; 2002. p. 27-36.

11 Whitehead M. The concepts and principles of equity in health. Int J Health Serv 1992; 22:429-445.

12 World Health Organization. Final report of the Commission on Social Determinants of Health. Closing the gap in a generation. Health equity through action on the social determinants of health. Geneva: WHO; 2008.

13 Sen AM. ¿Por qué la equidad en salud? Revista Panamericana de Salud Pública 2002; 11: 302-310.

14 UNICEF, Informe sobre Equidad en Salud 2016 Un análisis de las inequidades en salud reproductiva, materna, neonatal, de la niñez y de la adolescencia en América Latina y el Caribe para guiar la formulación de políticas.

15 US Department of Health and Human Services. Oral Health in America: A report of the surgeon general - Executive summary. Rockville, MD: US Department of Health and Human Services, National Institute of Dental and Craniofacial Research, National Institutes of Health; 2000.

16 Slade GD, Spencer AJ, Roberts-Thomson KF. Australia's dental generations: The National Survey of Adult Oral Health 2004-06. Canberra: Australian Institute of Health and Welfare; 2007

17 Pizarro V, Ferrer M, Domingo-Salvany A, Benach J, Borrell C, Pont A, Schiaffino A, Alamnza J, Tresserras R, Alonso J. The utilization of dental care services according to health insurance coverage in Catalonia (Spain). Community Dent Oral Epidemiol. 2009; 37: 78-84.

18 European Commission. Health in Europe, Results from 1997-2000 surveys. Theme 3, Population and social conditions. Luxembourg: Office for Official Publications of the European Communities; 2003

19 Rev. Gerenc. Polit. Salud, Bogotá (Colombia), 12 (25): 96112, julio-diciembre de 2013.

20 Organización Mundial de la Salud. Las inequidades provocan una enorme mortandad, según el informe de una comisión de la OMS. Ginebra: OMS; 2008

21 Hernández-Vásquez A, Azañedo D, Díaz-Seijas D. y cols. Acceso a servicios de salud dental en menores de doce años en Perú, 2014. 2016;12(3):429-441

22 Ministerio de Salud del Perú. Plan de Salud Escolar 2015. Disponible en URL:

23 Azañedo D, Diaz-Seijas D. Salud oral del adulto mayor: acceso, desafíos y oportunidades para la salud pública peruana. Rev. Peru Med Exp Salud Pública. 2016; 33(2):3735.

24 Ministerio de Salud del Perú. Dirección General de Salud de las Personas. Plan de Intervención para la Rehabilitación Oral con Prótesis Removible en el Adulto Mayor 2012-2016 "Vuelve a Sonreir". Lima: MINSA; 2012.

25 Academia Peruana de Salud. Historia de la Salud en el Perú. Sistema nacional de salud. Lima: Academia Peruana 
de Salud; 2008.

26 Academia Peruana de Salud. L Foro "Salud y Desarrollo". El futuro de la salud en el Perú. Rev Acad Peru Salud. 2013;20(1):30-60.

27 Sánchez-Moreno F. Reforma del sistema nacional de salud y seguridad social universal. Exposición en la Comisión de Salud y Población del Congreso de la República. Rev Acad Peru Salud. 2011;18(2):4-14.

28 Academia Peruana de Salud. Historia de la Salud en el Perú 2007-2011. Salud y desarrollo. Lima: Academia Peruana de Salud; 2013

Recibido: 08 de Diciembre de 2017.

Aceptado: 28 de Diciembre de 2017. 
